\title{
STÉRÉOTYPES NATIONAUX ET CRÉATION ROMANESQUE DANS À LA RECHERCHE DU TEMPS PERDU
}

\begin{abstract}
A bstract. Fraisse Luc, Stéréotypes nationaux et création romanesque dans $\propto \dot{A}$ la Recherche du temps perdus [National stereotypes and literary creation in $\dot{A}$ la recherche du temps perdu]. Studia Romanica Posnaniensia, Adam Mickicwicz University Press, Poznań, vol. XXX: 2003, pp. 189-207. ISBN 83-232-1270-8. ISSN 0137-2475.

In his novel cycle $\dot{A}$ la recherche du temps perdu (1913-1927), Marcel Proust takes up a long-lasting process of psychological analysis carried on in the first person. This person represents, in a way, human nature and the reader's thought as well. Therefore, it seems that the question of national stereotypes should not have found its place in this kind of work, in which the fundamental psychological project did not allow the author to caricature national particularisms. Yet, they do find their incarnations in the whole series of characters (Poznan archives enable us to show even the hidden source of one of them) in order to subject this stereotypical vision to analysis and more profound criticism. What is peculiar, however, is that in the final analysis, prejudices against foreign nations are positively re-assessed because they help to understand the nature of the novelist's vision and literary activity.
\end{abstract}

Pourquoi un écrivain mettrait-il en scène des stéréotypes nationaux? Quelle place les stéréotypes nationaux peuvent-ils recevoir dans la littérature? D'emblée, toute une série de réponses se présentent à l'esprit, et il faudrait brièvement les dénombrer d'abord pour comprendre la situation que va occuper l'entreprise romanesque de Proust par rapport à ces possibilités, à ces interrogations.

On s'attend en premier à rencontrer des étrangers stéréotypés au sein d'œuvres militantes, essentiellement patriotiques, dénonçant les ennemis héréditaires de la nation. L'écrivain est alors, pense-t-il, au service du pays, dont il épouse les causes, les dangers, le besoin de revanche. Ce type de littérature a pu se développer à la faveur de l'exaltation des nationalités au $\mathrm{XIX}^{\mathrm{e}}$ siècle, mais ce contexte historique n'a pas créé le phénomène: les Sarrasins de la Chanson de Roland en témoignent. Si même il n'entend pas défendre une telle cause collective, l'écrivain peut souhaiter, de façon déjà plus distanciée, refléter les mentalités de son temps; il met en cuvre la conception qui s'est développée autour de Mme de Staël, d'une lit- 
térature reflet de la société. Un reflet non nécessairement passif: les stéréotypes nationaux ouvrent à la littérature le riche champ de la satire, ou encore de la caricature. De là, ce sens critique peut refluer vers les idéologies nationales, et l'écrivain observera alors en surplomb la vision particularisante et déformée qu'une nation sc fait d'une autre nation. On sait que le relativisme mis en avant et pour ainsi dire systématisé par la philosophie des Lumières invite le lecteur de telles œuvres à considérer comment l'observateur se stéréotype lui-même en stéréotypant les autres.

Mais qu'elle soit critique ou non critique, la mise en scène de stéréotypes nationaux entre dans le champ propre de la création littéraire: la satire, la caricature de l'étranger obéissent aussi aux lois d'un genre. Au théâtre, l'étranger type occupera ce qu'on appelle un emploi. Dans le roman, il incarnera une sorte de personnage groupe, qui ne lui confère pas une grande profondeur psychologique. Par ailleurs, la présence de personnages stéréotypés dans une œuvre pose indirectement, mais néanmoins nettement, la question de la position de l'autcur: est-il de parti pris, recherche-t-il l'objectivité, adhère-t-il par conviction à la mentalité de ses contemporains, ou veut-il au contraire s'en faire le dénonciateur? Autant de questions que le lecteur va lui retourner, et qui vont l'obliger peut-être à abandonner ce que Barthes appelle, au moins dans la littérature contemporaine, le degré zéro de l'écriture.

L'entreprise romanesque de Proust entretient des relations diverses avec ces différentes questions. Sa conception philosophique de l'art et de l'écrivain ne lui permet pas de se considérer comme la voix de ses contemporains. Il est évident que la Recherche du temps perdu ne peut se définir en premier comme le roman de la nation française, regardant à distance, tour à tour avec sympathie ou hostilité, les autres nations, allemande, anglaise, italienne ou espagnole. L'Allemand lui-même, dans une telle perspective intemporelle, ne peut se réduire à l'ennemi héréditaire. Il est vrai que même un philosophe se trouve impliqué dans la vie de son temps: le temps de Proust, c'est la Grande Guerre, et de fait, les pages du Temps retrouvé évoquant le premier conflit mondial font malgré tout entendre cette voix nationaliste issue en France de la guerre de tranchées. Mais de plus, ce qui peut embarrasser un romancier tel que Proust, dans la mise en scène de stéréotypes nationaux, c'est ce que cette simplification retire de psychologie aux personnages: dans une narration dont la systématique élucidation psychologique constitue l'un des principaux ressorts, que faire d'un personnage affublé d'une simple étiquette, celle qui porte le nom de son pays d'origine? Dans une œuvre qui travaille sans cesse à la différenciation des nuances les plus fines, que faire de cet étiquetage qui rend seulement compte des idées reçues que nourrissent les nations les unes sur les autres? Il y a de plus, dans la réduction d'une personne à un type, et à un type caricaturé, donc moqué d'avance, une cruauté à laquelle ne pourra jamais se résoudre un Proust.

Et pourtant, les étrangers stéréotypés ne manquent pas, dans l'appareil des personnages proustiens. Cela est inévitable dans cette nouvelle Comédie humaine qui comporte un si grand nombre de figures diversifiées; et aussi dans une ouvre contemporaine de la Belle Époque, au cours de laquelle tant de représentants de na- 
tions étrangères sont venus exposer dans la capitale française leur exotisme relatif. Écrivain reclus qui n'a presque jamais voyagé à l'étranger, Proust ne se trouvait guère en situation d'observer par lui-même les particularités, comiques ou irritantes, ou simplement curieuses, de ce qui n'est pas français; son époque remédie cependant à cette lacune biographique, en faisant affluer l'étranger de toutes nations dans la société mondaine qui fut à peu près son seul milieu. J'ai étudié ailleurs l'évocation pittoresque par Proust de ces souverains étrangers de passage ou en résidence à Paris, dont les figures se reflètent dans sa correspondance privée puis dans son cuvre romanesque ${ }^{1}$. Les étrangers typés ne seront donc pas rares dans la Recherche $d u$ temps perdu, parce que dans cette cuvre, comme le confie le romancier dès avant la parution de $D u$ côté de chez Swann, «il y a un monsieur qui raconte et qui dit: Je; il y a beaucoup de personnages» ${ }^{2}$. Mais précisément, ce monsieur qui dit: je n'est pas Proust; il ne représente pas le Français de la Belle Époque, qui observe l'Allemand ou l'Italien mais, selon l'esprit philosophique du temps, en quelque sorte la nature humaine elle-même à laquelle il prête sa voix: face à lui, les autres personnages sont les objets d'une élucidation psychologique, non les caricatures d'un esprit national ou communément considéré comme tel.

C'est cette concomitance d'une présence et d'une aporie des stéréotypes nationaux dans l'entreprise romanesque de Proust qui est susceptible de présenter de l'intérêt. Pour tâcher de démêler cet écheveau, l'analyse peut aller de la vie à l'œuvre, c'est-à-dire aussi de la société à la création, en observant donc d'abord la présence, dans l'esprit et sous la plume de Proust, des stéréotypes nationaux, pour retrouver ensuite les explications que le romancier propose de cette vision stéréotypée, puis la critique sous-jacente qu'il soulève à son encontre dans son œuvre, et enfin, surplombant ce cheminement critique, l'éloge paradoxal des stéréotypes nationaux qui se justifieront dans la mesure où ils peuvent servir et même donner à comprendre une entreprise romanesque et la création littéraire.

\section{***}

Le psychologue des profondeurs et le philosophe de la mémoire involontaire n'excluent pas en Proust un homme sujet aux idées reçues sur les nations étrangères. Ces idées reçues seront mises en scène, de nation en nation, par des chemins très divers; mais elles ont pour socle commun la célébration de la civilisation française, qui dans la Recherche du temps perdu porte un nom: Saint-André-desChamps. Cette église perdue dans la campagne de Combray, dont la situation évoque fortement celle de Saint-Éman à côté d'Illiers, à cause des statues que le roman-

\footnotetext{
1 «Têtes couronnées dans la correspondance de Proust» (Les grands hommes des autres, Actes du $X^{\mathrm{c}}$ colloque Poznań-Strasbourg des 4-6 novembre 1998, publiés sous la direction de Maciej Serwański, Poznań, Instytut Historii UAM, 2000, pp. 261-274).

${ }^{2}$ Correspondance de Marcel Proust, édition établie, présentée et annotée par Philip Kolb, Paris, Plon, 21 vol., 1970-1993; t. XII, p. 92.
} 
cier sculpte à son porche, représente la simplicité, le courage et la naïveté des petits Français dont l'esprit peut survivre aujourd'hui depuis le moyen âge. Le héros et narrateur du roman se montre d'emblée à l'unisson de cette tradition populaire: «Souvent aussi nous allions nous abriter, pêle-mêle avec les Saints et les Patriarches de pierre sous le porche de Saint-André-des-Champs. Que cette église était française!» ${ }^{3}$. Ce qu'est cette église française, la domestique Françoise l'incarne, avec son franc-parler pittoresque et son bon sens populaire, que le baron de Charlus retrouve dans les rues de Paris en guerre - nous voici done à l'époque du Temps retrouvé -, lorsqu'il désigne du geste les «petits Parigots, tenez, comme celui qui passe là, avec son air dessalé, sa mine éveillée et drôle» ${ }^{4}$; mais aussi d'autres personnages du roman, comme Albertine: «Albertine - et c'était peut-être, avec une autre que l'on verra plus tard, une des raisons qui m'avaient à mon insu fait la désirer - était une des incarnations de la petite paysanne française dont le modèle est en pierre à Saint-André-des-Champs» ${ }^{5}$. Domesticité, bourgeoisie ici, noblesse aussi, car c'est à propos du marquis de Saint-Loup que cet esprit français est défini avec le plus de généralité: «le véritable opus francigenum, dont le secret n'a pas été perdu depuis le XIII siècle, et qui ne périrait pas avec nos églises, ce ne sont pas tant les anges de pierre de Saint-André-des-Champs que les petits Français, nobles, bourgeois ou paysans, au visage sculpté avec cette délicatesse et cette franchise restées aussi traditionnelles qu'au fameux porche, mais encore créatrices» ${ }^{6} . \mathrm{Ce}$ fonds français, que composent un petit nombre seulement de caractéristiques, le romancier en a trouvé les éléments en lisant les ouvrages de l'historien de l'art chrétien Émile Mâle, dont le tout premier livre, issu de sa thèse en 1898 , L'Art religieux du XIII siècle en France, met en équation deux apogées des cathédrales médiévales, dans le temps au XIII ${ }^{\mathfrak{e}}$ siècle, et dans l'espace en France.

Face à ces qualités bien françaises, diverses nations apparaissent chacune bien typée, selon un jugement sous-entendu qui va de la sévérité à l'indulgence. La seule dureté que semble prendre à son compte le narrateur de la Recherche touche une ambassadrice de Turquie, que le héros rencontre chez la duchesse puis la princesse de Guermantes, et qui sous sa plume parle «avec un peu de bassesse et de sensualité orientales», mais surtout - ce qui adoucit la charge en trait humoristique sur les étapes de l'apprentissage mondain - appelle Babal Hannibal de Bréauté, et Mémé Palamède de Charlus qu'elle ne connaît ni l'un ni l'autre, «à cause d'une instruction trop rapide qui lui faisait nommer ces nobles seigneurs selon ce qu'elle croyait la coutume du pays. Elle avait fait ses classes en quelques mois et n'avait pas suivi la filière»?

${ }^{3} \dot{A}$ la recherche du temps perdu, édition réaliséc sous la direction de Jean-Yves Tadié, Paris, Gallimard, «Bibliothèque de la Pléiade», 4 vol., 1987-1989, t. 1, p. 149.

${ }^{4}$ Id., t. IV, p. 386.

${ }^{5}$ Id., t. II, p. 662.

${ }^{6}$ Ibid., p. 703.

${ }^{7}$ ld., 2. 1II, pp. 59-60. 
Dans l'immédiat après-guerre, c'est avec un amusement médusé que Proust observe ce qu'il considère comme des excentricités chez les Américains, rendant compte dans une lettre de la vie fastueuse de l'industriel Rockefeller (1839-1937) ${ }^{8}$, ou rapportant cette anecdote, sur une riche Américaine de Chicago: «À propos de Mr Channon, j'ai reçu une lettre de sa mère me demandant mes livres pour une bibliothèque qu'elle a fondée, à Chicago. C'est très naturel et gentil; la fin seule m'a surpris: 'Croyez à mes respectueux souvenirs'. Respectueux m'a fait supposer que vous aviez inculqué à chacun l'idée que j’avais personnellement connu JeanJacques Rousseau et peut-être Bossuet. Souvenirs ne m'a pas moins étonné car je ne connais pas cette dame. [...] Son prénom est inattendu et charmant: c'est "Vesta'»".

Face à la richesse américaine, la vieille Russie ne peut être, en ce début du $\mathrm{XX}^{\mathrm{c}}$ siècle, que hautement titrée: dans la Recherche, la princesse Sherbatoff représente la "grande dame russe», «brouillée avec sa famille, exilée de son pays» ${ }^{10}$, une telle position correspondant à celle d'une contemporaine de Proust, la princesse Gortchakoff, disparue en $1905^{i l}$. Plus tard, le romancier s'estimera rattrapé par la vie (ce qu'il trouve, dit-il, «très Goncourt») en 1915, en lisant dans les journaux «que le Tzar a pris comme Ministre le Prince Sherbatof [Tcherbatof en fait] (la princesse Sherbatof remplit le troisième volume» de son cycle romanesque ${ }^{12}$, ainsi qu'il l'explique amusé à un correspondant). En parallèle cette fois à la richesse excentrique des Américains, il faut noter que le monde slave fait des apparitions comiques, dans la société des années 20; en témoigne au besoin cette lettre de 1922 à la princesse Soutzo qui deviendra Mme Paul Morand: «Dans l'excellente Revue de fin d'année où, compère agoraphobique et chancelant, j'entrais plus mal que bien [...], vous ne m'avez pas laissé vous dire bonjour et vous m'avez entraîné vers Mme Landowska [pianiste et compositeur polonais] en train de mordre Mlle Vacaresco à la fesse»; Proust contemplateur de cette étonnante scène n'est lui-même pas moins comique, «dans [son] trouble et près de [son] tilleul» ${ }^{13}$.

Les nations les plus proches, connues plus fréquemment, font de ce fait l'objet des simplifications les plus marquées. L'Angleterre se résume ainsi à l'élégance de Brummel (1778-1840), qui apparaît dans des esquisses des Jeunes filles en fleurs puis de Sodome et Gomorrhe, «le roi des élégances» ${ }^{14}$. Mais la vraie élégance anglaise est incarnée par un ambassadeur victorien, lord Lytton (1831-1891), «qui après avoir été Vice-Roi des Indes était à ce moment Ambassadeur d'Angleterre à Paris», et amoureux de Mme Straus, ce qui inspire à Proust épistolier ce portrait:

\footnotetext{
${ }^{B}$ Correspondance, t. XVIII, p. 178.

${ }^{9}$ Id., t. XVII, p. 323.

${ }^{10}$ Recherche, t. III, pp. 269-270.

"Correspondance, $\mathrm{t}$. XI, p. 63 et note 23.

${ }^{12}$ ld., t. XIV, p. 202.

${ }^{13}$ Id., t. XXI, p. 58.

${ }^{14}$ Recherche, t. I, p. 1347; t. III, pp. 983 et 996.
} 
«Cet homme, exquis d'ailleurs, venait la voir tous les jours, et nous allions tous nous promener. Sa magnifique calèche de l'Ambassade, ses beaux chevaux et ses cochers aussi grands que lui, ne me donnaient aucune impression d'élégance. Mais un jour que nous avions été avec cette dame nous promener en tramway ce qui était notre grand plaisir, le dit Lytton montant avec nous, se fait suivre pendant une heure de la calèche vide, qui s'arrêtait aux stations et quand nous 'changions' aux 'correspondances'. Le cocher moins grand seigneur que son maître était furieux. Alors j'ai eu une impression d'élégance»" ${ }^{15}$. Mais après la guerre, on dirait que même l'élégance anglaise est contaminée par cette excentricité qui semble avoir gagné toute la société parisienne, comme on le voit chaque fois que l'écrivain découvre au Ritz, vers la fin de la guerre, les représentants officiels de l'Angleterre à Paris: «J' ai été dîner, ce soir, seul au Ritz [...]. Le directeur de l'établissement [...] m'a appris qu'à la table voisine il y avait Winston Churchill et sa suite. [...] Je ne sais plus quel Malborough [cousin germain de Churchill] avait une énorme cravate blanche, ce qui était aussi diplodocus que le haut de forme de Lord Bertie [ambassadeur de Grande-Bretagne en France]. Mais quand ces choses sont faites volontairement, on a l'air, au lieu d'un notaire de village, d'un beau portraits ${ }^{16}$. C'est finalement un éloge.

Face à l'Anglais élégant, l'Allemand est certes lourd, mais la poésie des noms lui permet de tirer son épingle du jeu des ridicules nationaux: tel apparaît, dans la Recherche (comment ne pas songer au baron de Thunder-ten-tronckh dans Candi$d e$ ?), le prince de Faffenheim-Munsterburg-Weinigen, rhingrave et électeur palatin, premier ministre allemand, qui fait son apparition dans le salon de Mme de Villeparisis: «Le nom du prince gardait, dans la franchise avec laquelle ses premières syllabes étaient - comme on dit en musique - attaquées, et dans la bégayante répétition qui les scandait, l'élan, la naïveté maniérée, les lourdes 'délicatesses' germaniques projetées comme des branchages verdâtres sur le «Heim» d'émail bleu sombre qui déployait la mysticité d'un vitrail rhénan derrière les dorures pâles et finement ciselées du XVIII siècle allemand" " ${ }^{17}$. Le prince allemand, dont la femme est bien sûr - stéréotype oblige - à la tête de la coterie la plus fermée de Berlin ${ }^{18}$, se signale au héros par sa raideur, puisque «pour le malheur de mes phalanges qui n'en sortirent que meurtries, je les laissai s'engager dans l'étau qu'était une poignée de main à l'allemande»" ${ }^{19}$, et il prononce évidemment archéologue «arshéologue»: son langage est retranscrit comme chez Balzac celui de Nucingen ${ }^{20}$.

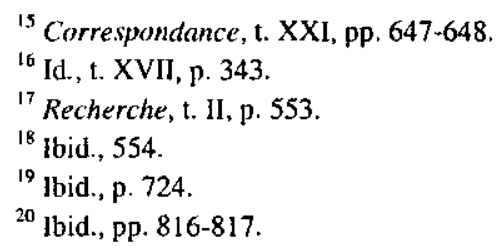


Cette présence souriante des stéréotypes nationaux, sous la plume de Proust épistolier puis romancier. connaît du moins dans l'œuvre un certain nombre d'explications théoriques qu'il n'est pas inintéressant de prendre en compte, si l'on veut nourrir une réflexion sur ce sujet. Une telle vision simplifiée participe du premier âge de la conscience, tant individuelle que collective, que le romancier dogmaticien, sclon le modèle des trois âges distingués par Auguste Comte, met en scène - pour être ensuite dépassé - dans les premiers volumes de son cycle romanesque, et qu'il nomme l'âge des noms ou des croyances. Le stéréotype serait en somme d'abord le produit d'un imaginaire enfantin, chez celui qui n'a encore jamais quitté sa première sphère de vie, tel le hérus qui va apercevoir pour la première fois la princesse de Parme: «À défaut d'être encore jamais de ma vie allé à Parme [...], en connaître la princesse qui, je le savais, possédait le plus beau palais de cette cité unique où tout d'ailleurs devait être homogène, isolée qu'elle ćtait du reste du monde, entre les parois polies, dans l'atmosphère, étouffante comme un soir d'été sans air sur une place de petite ville italienne, de son nom compact et trop doux, cela aurait dû substituer tout d'un coup à ce que je tâchais de me figurer, ce qui existait réellement à Parme, en une sorte d'arrivée fragmentaire et sans avoir bougé» ${ }^{21}$. Sinon que l'imaginaire de l'enfant futur écrivain diversifie son stéréotype en vision poétique, alors que les peuples simplifient les leurs en une caricature figée, dont la production ressortit à ce même âge primitif de la conscience, et que le narrateur caractérise ainsi: "Ces images étaient fausses pour une autre raison encore; c'est qu'elles étaient forcément très simplifiées ${ }^{22}$.

Mais le stéréotype national, qui interdit au personnage typifié d'être une personne, illustre encore ce principe du narrateur, selon lequel «notre personnalité sociale est une création de la pensée des autres» ${ }^{23}$. Maxime applicable à un petit cercle, mais aussi bien à la vie internationale: notre personnalité nationale est une création de la pensée des autres nations.

Par là, l'humour conventionnel attaché au portrait de personnalités étrangères met le lecteur en présence d'un problème de philosophie générale, la connaissance que nous prenons de la réalité extérieure. Cette connaissance, nous dit Kant, doit en passer par les catégories de notre entendement; le monde, ajoute Schopenhauer sous un angle différent mais issu du premier, est ma représentation. Dès lors, le sujet ne peut faire autrement que de percevoir cet autre plus autre que les autres qu'est pour un Français moyen le Russe, l'Américaine ou l'Anglais, selon les catégories, en nombre limité, de sa représentation.

Une explication assez complète de l'élaboration des stéréotypes nationaux dans une pensée collective était enfin fournie à Proust - et peut nous être encore foumie

\footnotetext{
${ }^{21}$ Ibid., p. 719.

${ }^{22}$ Id., t. J, p. 382.

${ }^{23}$ Ibid., p. 19.
} 
aujourd'hui - par un sociologue contemporain qui informe souterrainement sa société romanesque: Gabriel Tarde théoricien de l'imitation. Selon ce sociologue rival d'Émile Durkheim, qui a développé sa théorie essentiellement dans Les Lois de l'imitation et La Logique sociale ${ }^{24}$, le comportement des individus en société est régi par une loi, chez eux inconsciente, d'imitation: pour résumer, l'homme imite son passé par la tradition, son présent par la mode, et lui-même par l'habitude. L'évolution sociale est faite de l'invention d'un petit nombre de créateurs, qui est ensuite copiée peu à peu par le grand nombre. C'est ce qui oppose Tarde à Durkheim - pour qui le fait social s'impose à l'individu de l'extérieur par la collectivité -, à savoir que l'état social n'est qu'un état psychologique élargi par contamination: «la marche de l'imitation» se fait «du dedans au dehors»; pour imiter un comportement social, il faut chez l'individu une adhésion psychologique préalable ${ }^{25}$. «Pour qu'une bonne combinaison d'idées éclaire les esprits d'une nation, il faut qu'elle luise d'abord dans un cerveau isolé» ${ }^{26}$. Ce qui veut dire - c'est essentiel pour un romancier - que «le psychologique s'explique par le social, précisément parce que le social naît du psychologique» ${ }^{27}$.

Par le fruit de cette imitation inconsciente, l'individu vit semblablement sous l'influence de son milieu familial, professionnel ou national. Ce qui veut dire que les relations entre nations n'est qu'une psychologie collective: deux nations face à face ne sont au fond qu'un imité et un imitateur. C'est ce qui permet à Gabriel Tarde de définir «la diplomatie, cette politesse des États. Par la diplomatie, les orgueils nationaux apprennent à se manier ou à se masquer pour s'accorder en apparence, de même que, par la politesse, les orgueils individuels ou domestiques se dissimulent les uns aux autres et se flattent réciproquement ${ }^{28}$. En fait, ce que le sociologue appelle «la logique sociale» veut qu'en permanence, une idée novatrice soit en lutte avec une jdée admise, dans un «duel logique». L'idée neuve peut être l'influence récente d'un peuple sur un autre; l'idée admise, l'influence ancienne d'un peuple sur un autre. C'est par là que la logique sociale se trouve au cœur de notre sujet, car elle s'observe aisément à travers le comportement d'un peuple à l'égard d'un autre. Notamment, l'hostilité d'une nation pour une autre signale, contrairement à ce qu'on pourrait d'abord croire, une prochaine imitation, un prochain passage sous influence: «Partout et toujours, il est remarquable que les nations closes, avant de se connaître, commencent par se mépriser les unes les autres, parfois même par se haïr» ${ }^{29}$; mais prenons-y garde: «le moment où deux peuples, jusque-là indifférents l'un à l'autre, se mettent à éprouver de l'antipathie l'un pour

\footnotetext{
${ }^{24}$ Alcan, 1890 et 1894: pour les deux ouvrages, nous utilisons la ré́dition de 1895.

${ }^{25}$ Les Lois de l'imitation, p. 223.

${ }^{26}$ Ibid., p. 163.

${ }^{27}$ Ibid., préface à la deuxième édition, p. VIII, note.

${ }^{28}$ La Logique sociale, p. 336.

${ }^{29}$ Ibid., p. 298.
} 
l'autre, est celui où ils commencent à s'imiter réciproquement» ${ }^{30}$. Dans ce contexte, la guerre est considérée comme le «type le plus net du duel logique social» ${ }^{31}$, donc entre une valeur dominante mais en déclin et une valeur montante; mais il faut aussi $\mathrm{s}$ 'attendre à une imitation involontaire de l'ennemi à la faveur des guerres ${ }^{32}$.

C'est ce contexte de pensée qui a poussé toute une génération à concevoir la vision d'une nation par une autre nation comme une psychologie dont on entendait détailler les lois, ces lois étant essentiellement l'imitation et la contre-imitation. Phénomène à la fois subtil et grossier: à la même époque, en 1895, Gustave Le Bon prenait en compte ce jugement d'un peuple par un autre dans sa $P$ sychologie des foules (Alcan): sous la plume de le Bon, la psychologie des foules est synonyme de simplification primaire, d'illusion grossière, de conviction mobile aussi: «Les raisonnements des foules sont toujours d'ordre très inférieur. Les idées qu'elles associent n'ont que des apparences d'analogie ou de succession» ${ }^{33}$. Un romancier comme Proust trouvait ici matière à mettre en scène cette psychologie collective grossière, qui applique un ou deux qualificatifs tout au plus au représentant de chaque nationalité; mais Tarde l'incitait malgré tout à entrer plus avant dans les arcanes de cette psychologie, faite de refus et d'acceptations superposés. C'est ce qui apparaîtra mieux, après ces diverses explications des stéréotypes nationaux, dans le développement de leur critique.

L'unjvers de Combray, sur lequel s'ouvre le regard du héros de la Recherche du temps perdu, est un univers dépourvu de la perception de tout clivage, aussi bien social que national. L'idée est certes que ce paradis doit avoir une fin; mais dès lors, une critique sous-jacente parcourt sa société romanesque, petite volière cosmopolite, à l'image de la société 1900 puis 1920 , dans laquelle tout ce qui est étranger est à la fois cultivé et méprisé.

Si les stéréotypes nationaux, ainsi qu'on l'a vu, ont bonne presse dans l'univers de la Recherche, il faut ajouter que Proust met volontiers en échec ce type d'idées reçues, d'une façon d'ailleurs subtile qui consiste à montrer que, sous la nationalité caricaturée, il faut bien souvent en trouver une autre. Son narrateur lui-même ne prend jamais en charge ces caricatures, ce que montrent mieux que tout ses brouillons. Ainsi, pour le prince allemand caricaturant la raideur germanique, une esquisse révèle que le romancier avait en fait pensé auparavant l'appeler le prince Tchiguine $^{34}$ : son origine était dès lors Saint-Pétersbourg au lieu de Berlin. L'amour de

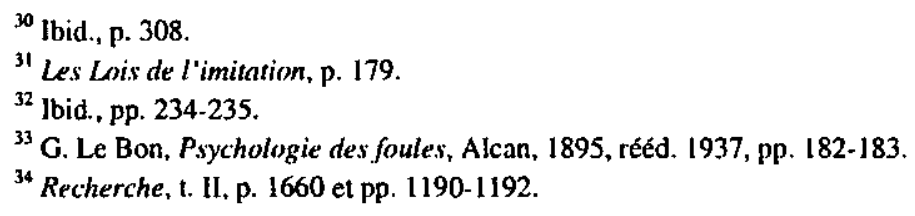


Swann pour Odette de Crécy bouleverse à plus d'un titre les stéréotypes: ainsi, la cocotte parisienne, ressemblant à la Zéphora de Botticelli, est donc une ouvre florentine $^{35}$. La passion jalouse de Swann fait passer sa cruauté tout asiatique au filtre d'un biographe italien: «Et Swann sentait bien près de son cœur ce Mahomet II dont il aimait le portrait par Bellini et qui, ayant senti qu'il était devenu amoureux fou d'une de ses femmes, la poignarda afin, dit naïvement son biographe vénitien, de retrouver sa liberté d'esprit» ${ }^{36}$. Mahomet serait en effet tombé amoureux d'une esclave, Irène, qu'il fit décapiter devant ses soldats, pour préserver la grandeur de sa maison; le chroniqueur vénitien est Giovanni Maria Angiolello (1451-1525), auteur d'une Historia turchesca, restée inédite jusqu'en $1909^{37}$. Ce que recherche le romancier en ménageant, autour de ses personnages, ces circonstances, c'est à provoquer des chassés-croisés de points de vue: c'est ainsi que le roi Théodose, dont on attend la visite à Paris dès la fin de $D u$ côté de chez Swann ${ }^{38}$, n'est finalement rien moins qu'un souverain oriental d'origine bavaroise: le marquis de Norpois l'a connu «à la cour de Bavière, quand il ne songeait pas à son trône oriental (vous savez qu'il y a été appelé par un congrès européen, et il a même fort hésité à l’accepter, jugeant cette souveraineté un peu inégale à sa race, la plus noble, héraldiquement parlant, de toute l'Europe)» ${ }^{39}$. Même littérairement parlant, ce que le narrateur des Jeunes filles en fleurs appelle le côté Dostö̈evski de Mme de Sévigné ${ }^{40}$ fait mentir les particularismes nationaux du Roman russe dont la vogue avait été créée en 1886 par le livre de Vogüé.

À ce stade, il est évident que le romancier du Faubourg Saint-Germain a beau jeu de recourir aux origines multinationales des aristocrates qu'il met en scène pour battre en brèche les stéréotypes que l'on pourrait rechercher en eux. C'est un fait que l'aristocratie rend impossibles les stéréotypes nationaux. Voici par exemple la princesse de Parme «alliée à toutes les familles royales de l'Europe», si bien qu'elle est aussi peu parmesane et stendhalienne «que, par exemple, à Paris, dans le quartier de l'Europe, la rue de Parme, qui ressemble beaucoup moins au nom de Parme qu'à toutes les rues avoisinantes, et fait moins penser à la Chartreuse où meurt Fabrice qu'à la salle des pas perdus de la gare Saint-Lazare»" ${ }^{4 !}$. Le grand-duc de Hesse, évoqué par le prince de Faffenheim, amène ces précisions, qui n'ont plus rien de germanique, de la part du duc de Guermantes: «son frère avait épousé ma sœur, $[\ldots]$ et d'ailleurs sa mère était cousine germaine de la mère d'Oriane» ${ }^{42}$. Le duc de Guermantes peut donc affirmer: «nous sommes du même sang que les Hes-

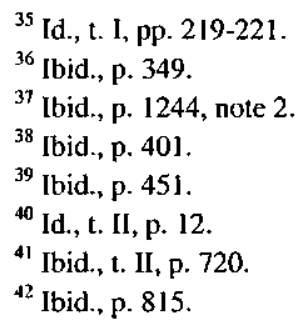


se», et à l'inverse «le grand-père du roi de Suède cultivait la terre à Pau» ${ }^{43}$. Le lecteur voit encore apparaître, plusieurs fois dans le cycle romanesque, Alexandra de Danemark, reine d'Angleterre ${ }^{44}$, ou Louise-Marie d'Orléans, reine des Belges ${ }^{45}$ et enfin Élisabeth, duchesse de Bavière, nièce de la reine de Naples, qui épousera le prince Albert de Belgique, futur Albert $I^{\mathrm{er}}$. Jongler ainsi avec les nationalités a nécessité, de la part du romancier, une documentation tournée en ce sens. Il s'est penché par exemple sur le cas de la maison de Dampierre, dont les aînés sont ducs de San Lorenzo ${ }^{46}$. À ce stade, son attention a été pour finir encore renforcée par la société cosmopolite que lui découvrent ses amis anglo-américains de l'après-guerre, tel Sydney Schiff, publiciste et romancier anglais, qui écrit à Proust au moment où il se prépare à un dîner, en 1921, en espérant pouvoir «parler avec $\mathrm{M}^{\text {rs }}$ Aldous Huxley, une Belge pas très intelligente, ou avec mon ami Michel de Zogheb, un Italo-Danois-Syrien qui est charmant ${ }^{47}$.

Dans le roman, le cas du baron de Charlus représente par-dessus tout ce mélange des nationalités en un seul individu qui pulvérise les stéréotypes. Pour accentuer sa démonstration, le romancier met en scène son personnage, au début du Temps retrouvé, durant la Grande Guerre qui exalte précisément les jugements à l'emporte-pièce sur les mentalités étrangères. Car dans ce contexte, le baron de Charlus, qui avait «eu une mère duchesse de Bavière» (comme son frère, le duc de Guermantes), «était par conséquent du corps-France comme du corps-Allemagne»; "n'étant pas véritablement français, il vivait en France» ${ }^{48}$; sa germanophilie s'affirme à l'occasion de la guerre: «il gémissait de la défaite des Allemands, parmi lesquels il se comptait» ${ }^{49}$. Il est à noter que Proust ne présente pas une telle attitude comme une trahison de l'esprit national, ni comme un réquisitoire pur et simple contre cet esprit national, mais comme un point de vue pluraliste incitant à une réflexion sur la fermeture ou la déformation du jugement que provoque la simplification des mentalités sous l'effet - la doctrine de Gabriel Tarde n'est pas loin - d'une crise collective contemporaine. Car la finesse aristocratique du baron de Charlus, ajoutée à l'internationalisme de ses origines, le détache de tout stéréotype national, même au cour du conflit, à la faveur de ce regard dialectique: «Il était très fin, et les sots sont en tout pays les plus nombreux; nul doute que, vivant en Allemagne, les sots allemands défendant avec sottise et passion une cause injuste ne l'eussent irrité; mais, vivant en France, les sots français défendant avec sottise et passion une cause juste ne l'irritaient pas moins» 50 .

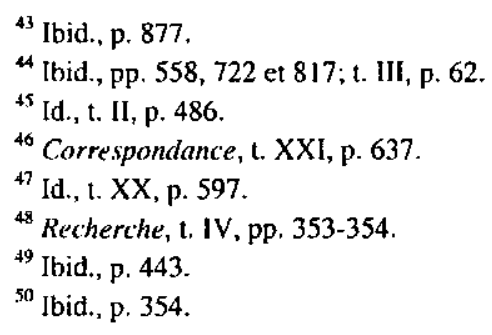


Ces exemples montrent que les stéréotypes nationaux, présents dans le cycle romanesque, constituent non pas un objet d'adhésion, mais bien plutôt un objet d'expérience. Deux cas le prouvent encore, intéressants à considérer dans la mesure où les personnages en question, qui n'ont pas de nom, sont désignés simplement par leur activité et leur nationalité: ne doivent-ils pas dès lors résumer en eux le pays d'où ils viennent? Nullement en fait: quand apparaît chez le prince de Guermantes «un musicien bavarois à grands cheveux» ${ }^{51}$, le détail descriptif s'applique davantage au musicien qu'au Bavarois; et quand apparaît, à la Raspelière, «un illustre philosophe norvégien qui parlait le français très bien mais très lentement», c'est parce qu'il est norvégien, mais surtout à l'usage parce qu'il est philosophe ${ }^{52}$; «le Scandinave", ainsi qu'on le désigne ${ }^{53}$, entretient en fait quelque rapport avec un philosophe français cousin de Proust: Bergson ${ }^{54}$. Le romancier avoue d'ailleurs dans une lettre que son «philosophe norvégien» a pour modèle direct le traducteur suédois de Bergson ${ }^{55}$ !

Reprenant toute une tradition de la littérature française, Proust ménage même dans son roman des épisodes qui reformulent la leçon des cannibales à la Montaigne et des Persans à la Montesquieu. Dans les Jeunes filles en fleurs, Swann raconte au héros, à la demande d'Odette, cette anecdote plaisante: «Vous savez que Mme Blatin aime à interpeller tout le monde d'un air qu'elle croit aimable et qui est surtout protecteur. $|\ldots|$ Elle est allée dernièrement au jardin d'Acclimatation où il y a des noirs, des Cinghalais, je crois, a dit ma femme qui est beaucoup plus forte en ethnographie que moi. [...] Enfin, elle s'adresse à un de ces noirs: 'Bonjour, négro!' [...] Ce qualificatif ne plut pas au noir: 'Moi négro, dit-il avec colère à Mme Blatin, mais toi, chameau!'» ${ }^{56}$. L'anecdote condense en fait plusieurs circonstances réelles. C'est d'abord en 1883 que se tint au jardin d'Acclimatation une «exposition» de Cinghalais et d'Araucaniens. L'Exposition universelle de 1889, qui était doublée d'une Exposition coloniale, développa la mode de ces «expositions». Une partie de l'esplanade des Invalides était occupée par des villages sénégalais, alfourou, pahouin, indien, etc. Cette même année, les Parisiens pouvaient contempler des Cosaques au jardin d'Acclimatation ${ }^{57}$. Mais une lettre de 1916 révèle que, sur ce fond de réalité contemporaine, Proust a assisté à un incident à l'origine de l'anecdote, durant son séjour de l'année précédente à Cabourg: visitant des blessés de guerre recueillis dans la station côtière, "comme j'apportais un jour des jeux de dames aux noirs (Sénégalais et Marocains) qui aiment beaucoup ce jeu, une dame très bête (il y

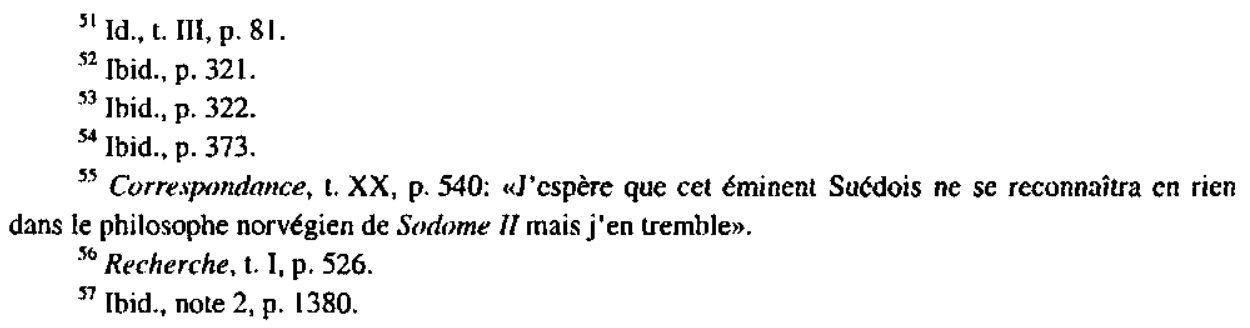

5. Correspondance, t. XX, p. 540: "J'cspère que cet éminent Sućdois ne se reconnaîtra en rien dans le philosophe norvégien de Sodome II mais j'en tremble».

${ }^{56}$ Recherche, t. I, p. 526.

$\$ 7$ Ibid., note 2, p. 1380. 
en a un nombre tout particulier à Cabourg) vint regarder ces noirs comme des bêtes curieuses et dit à l'un: 'Bonjour négro' ce qui le froissa horriblement. Il répondit: 'Moi négro mais toi chameau'»" ${ }^{58}$. Aux yeux de Proust, le rôle d'une transposition romanesque est visiblement de créer de tels renversements de situation: car le même épisode vécu inspire aussi le moment où le héros des Jeunes filles rencontre la princesse de Luxembourg sur la digue de Balbec: «dans son désir de ne pas avoir l'air de siéger dans une sphère supérieure à la nôtre elle avait sans doute mal calculé la distance, car, par une erreur de réglage, ses regards s'imprégnèrent d'une telle bonté que je vis approcher le moment où elle nous flatterait de la main comme deux bêtes sympathiques qui eussent passé la tête vers elle, à travers un grillage, au jardinn d'Acclimatation. Aussitôt du reste cette idée d'animaux et de bois de Boulogne prit plus de consistance pour moi. C'était l'heure où la digue est parcourue par des marchands ambulants et criards qui vendent des gâteaux, des bonbons, des petits pains. Ne sachant que faire pour nous témoigner sa bienveillance, la princesse arrêta le premier qui passa; il n'avait plus qu'un pain de seigle, du genre de ceux qu'on jette aux canards. La princesse le prit et me dit: 'C'est pour votre grand-mère'»" La circonstance cocasse, rappelée dans Le Côté de Guermantes: «j' avais vu la princesse de Luxembourg acheter des petits pains de seigle sur la plage pour en donner à ma grand-mère, comme à une biche du Jardin d'Acclimatation» ${ }^{60}$, fait refluer la réflexion critique à propos de l'exotisme des étrangers et des particularismes ethniques jusque dans les structures narratives, puisque dans cet épisode, le Sénégalais, c'est le narrateur lui-même, source du récit.

Une certaine tradition de la Renaissance et des Lumières s'insinue, ce qui est rare chez Proust, dans cette situation qui ridiculise le préjugé ethnique, qui caricature le stéréotype national. Comme chez Montaigne, comme chez Montesquieu, l'étranger est mis en scène en tant qu'il incarne une réplique inattendue à la vision commune; son origine plus ou moins lointaine garantit la nouveauté et la justesse de son point de vue. Le montre encore pour finir un épisode, parallèle à celui qu'on vient de voir, et dans lequel, chez le prince de Guermantes, Mme d'Arpajon est trempée par le jet d'eau d'Hubert Robert: «Alors non loin d'elle, un grognement scandé retentit assez fort pour pouvoir se faire entendre à toute une armée et pourtant prolongé par périodes comme s'il s'adressait non pas à l'ensemble, mais successivement à chaque partie des troupes; c'était le grand-duc Wladimir qui riait de tout son cœur en voyant l'immersion de Mme d'Arpajon [...]. Comme quelques personnes charitables faisaient remarquer au Moscovite qu'un mot de condoléances de lui serait peut-être mérité $[. .$.$] , le grand-duc, qui avait bon cœur, crut devoir s'exé-$ cuter et les demiers roulements militaires du rire à peine apaisés, on entendit un nouveau grondement plus violent encore que l'autre. 'Bravo, la vieille!', s'écriait-il

\footnotetext{
${ }^{58}$ Correspondance, t. XV, p. 45.

${ }^{59}$ Recherche, $\mathrm{t}$. II, pp. 59-60.

60 Jbid., p. 718.
} 
en battant des mains comme au théâtre» ${ }^{61}$. C'est-à-dire que le préjugé sur le grand personnage russe est aussitôt reflété en retour sur une représentante choisie de la société qui observe ces visiteurs étrangers et leur applique des épithètes toutes faites.

Présents dans le cycle romanesque, expliqués en théorie, caricaturés de façon critique, les stéréotypes nationaux sembleraient donc devoir être bannis de la $R e$ cherche. Encore faudrait-il ajouter qu'ils ne font pas seulement l'objet d'une dénonciation, d'une mise en scène distanciée et parée d'humour, sous la plume de Proust. Leur présence s'explique pour finir en ce qu'ils interfèrent dans l'entreprise romanesque. Quelles ressources ces stéréotypes offrent à un romancier installé dans son auvre, c'est ce qu'il importe d'examiner en dernier lieu.

On l'a aperçu à maints exemples, les stéréotypes nationaux concourent, par les voies les plus diverses, à la création des personnages. L'onomastique même lui permet de sérier en quelque sorte les personnages, les noms étrangers représentant la noblesse d'Empire, par opposition aux noms français représentant la noblesse d'Ancien Régime. Ainsi, à propos de la garmison de Doncières, qui doit beaucoup, dans la Recherche, à la ville d'Orléans où Proust a fait son volontariat, le romancier confie à un ami, au moment où paraît, en 1920, la première partie du Côté de Guermantes: «Je reconnais qu'il y a [...] un peu de Walewski opposé à Cholet dans Borodino» ${ }^{62}$; l'écrivain fait allusion à l'opposition, dans son souvenir, entre deux comtes, Armand-Pierre de Cholet et Charles Colonna Walewski, et dans la Recherche, entre le prince de Borodino et Robert de Saint-Loup. Il arrive à un personnage, qui subit une totale transformation du début à la fin de la Recherche, d'utiliser un stéréotype étranger pour parachever son changement d'identité: c'est ainsi l'anglomanie de Bloch, devenu, à la fin du Temps retrouvé, un écrivain reconnu sous le nom de Jacques du Rozier, qui permet ce changement: «Un chic anglais avait en effet complètement transformé sa figure et passé au rabot tout ce qui se pouvait effacer»; l'instrument de la métamorphose est bien sûr le monocle: «La part de machinisme que ce monocle introduisait dans la figure de Bloch la dispensait de tous ces devoirs difficiles auxquels une figure humaine est soumise, devoir d'être belle, d'exprimer l'esprit, la bienveillance, l'effort. La seule présence de ce monocle dans la figure de Bloch dispensait d'abord de se demander si elle était jolie ou non, comme devant ces objets anglais dont un garçon dit dans un magasin que 'c'est le grand chic', après quoi on n'ose plus se demander si cela vous plaît» ${ }^{63}$.

L'identité des personnages, c'est bien ce qui est en jeu, dans ce ballet de stéréotypes. Un cas très particulier le montre, celui des personnages qui, mieux que

\footnotetext{
${ }^{61}$ Ibid., t. Ill, p. 57.

${ }^{62}$ Correspondance, t. XIX, p. 460.

${ }^{63}$ Recherche, t. IV, pp. 530-531.
} 
le philosophe norvégien ou le musicien allemand, se voient dotés d'un nom abrégé, comme «le prince von» ou «le sculpteur Ski». Quel meilleur moyen de suggérer par la forme que le stéréotype est lui-même un abrégé de pensée? C'est le prince de Faffenheim, qui, en raison de la complexité pour un Français de ses trois noms, se voit communément surnommé le «prince Von» ${ }^{64}$. Une petite histoire peut d'ailleurs être reconstituée, derrière la création de ce personnage qui perd son nom en cours de roman. Philip Kolb, l'éditeur de la Correspondance de Marcel Proust, a pu établir que le modèle en est le prince Hugo von Radolin (1841-1917), ambassadeur d'Allemagne à Paris, que Proust commence à fréquenter à partir de $1905^{65}$. Mais ce que les lecteurs de Proust ne savent pas, c'est que cet ambassadeur allemand était en fait un Polonais de pure souche, sans doute le fils naturel de Bernard Potocki, luimême fils du romancier Jan Potocki, l'auteur de Manuscrit trouvé à Saragosse, comme le donnent à comprendre les lettres de Bernard conservées aux archives de Poznań. À l'origine, Hugo von Radolin s'appelait Radoliński! II importe dès lors de savoir que Proust connaît l'histoire de la maison Radolin par l'Almanach de Gotha, qui en donnait le récit entre celui de la maison de Putbus (nom d'un personnage de Sodome et Gomorrhe) et celui de la maison de Radziwił (famille amie du romancier). Ainsi le plus stéréotypé des Allemands de la Recherche est-il en fait polonais. Et dès lors, ce Radoliński devenu von Radolin dans la vie, et "prince von» dans l'œuvre, pourrait bien avoir donné le «ski» qui terminait son nom au sculpteur polonais «Ski», dont l'histoire est de son côté un peu semblable. Car le «sculpteur Ski, appelé ainsi à cause de la difficulté qu'on trouvait à prononcer son nom polonais»" ${ }^{66}$ 'appelle en fait Viradobetski ${ }^{67}$. Ici aussi, la correspondance de Proust nous livre quelques indices sur la formation de ce personnage énigmatique, dont la syllabe finale résume toute la Pologne, car en 1918, le romancier demande inopinément à une connaissance: «Savez-vous qui est un comte Hodorzsky (ou un nom de ce genre) ${ }^{68}$, que l'on ne trouve nulle part dans la société du temps; mais les contemporains pouvaient y reconnaître à d'autres traits Frédéric de Madrazo (18781938), peintre issu d'une famille espagnole - nouveau trait de déguisement par changement de nationalité.

Un autre trait du personnage proustien trouve à se nourrir des stéréotypes nationaux: sa théâtralité. On se souvient que le grand-duc Wladimir, encourageant Mme d'Arpajon aspergée par le jet d'eau chez le prince de Guermantes, battait des mains «comme au théâtre» ${ }^{69}$. Après le Voltaire de Candide, Proust se montre de même friand de s'instruire des différents modes étrangers de salutations (bien des

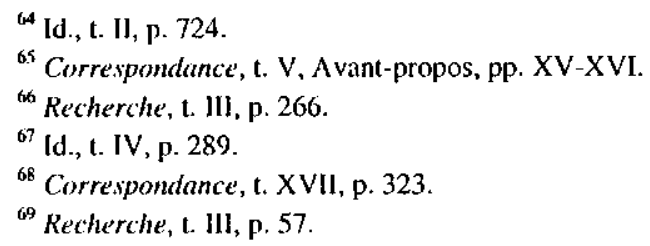


scènes de ce genre seront parsemées dans la Recherche). Après avoir lu Les huit $\mathrm{Pa}$ radis: Perse - Asie Mineure - Constantinople (Hachette, 1908) de la princesse $\mathrm{Bi}$ besco, Proust voudrait que l'auteur lui mime les salutations qu'elle évoque dans le passage suivant (nous sommes en Perse): «Tout en prenant nos sorbets à la rose, nous échangeons avec Son Altesse des compliments majestueux et puérils. 'Le plus beau jour de ma vie !' nous dit ce Prince à plusieurs reprises, en posant la main droite sur son cœur. Et nous répondons de même avec des salutations à n'en plus finir: 'C'est le plus beau jour de la nôtre!'»"

Ainsi le personnage de roman ne voit-il pas seulement son identité transformée ou affaiblie par le stéréotype dont il est le porteur; le pittoresque qu'il y puise lui donne aussi bien un singulier relief. Chez Proust, ce relief lui est conféré par le langage - et les stéréotypes nationaux sont fortement affaire de langage. L'anglomanie d'Odette devenue Mme Swann, ranimée par la guerre, lui fait dire: «Mon gendre Saint-Loup connaît maintenant l'argot de tous les braves tommies, il sait se faire entendre de ceux des plus lointains dominions et, aussi bien qu'avec le général commandant la base, fraternise avec le plus humble private ${ }^{71}$. Bien plus, cette anglomanie de langage, que l'on prend longtemps pour du simple snobisme, se révèle un jour la résurgence d'un fait biographique secret chez le personnage, mentionné dès le premier volume au moment où Swann jaloux avait mené sur elle son enquête: «ne disait-on pas que c'était par sa propre mère qu'elle avait été livrée, presque enfant, à Nice, à un riche Anglais?» ${ }^{72}$ (I, 361). Dans Le Temps retrouvé fleurissent maintenant les expressions de Brichot dans ses articles de guerre: «les Allemands ne pourront plus regarder en face la statue de Beethoven; Schiller a dû frémir dans son tombeau; Lénine parle, mais autant en emporte le vent de la steppe»; aux yeux du narrateur, ce sont autant d' «images qui ne voulaient rien dire du tout» ${ }^{73}$. Ailleurs cependant, le narrateur linguiste relève les simplifications que font subir symétriquement à leurs noms respectifs les Anglais et les Français, visibles dans la façon qu'ont les Guermantes de «prononcer les noms propres d'une façon aussi différente de leur orthographe que certains noms français quand ils sont proférés par les Anglais qui prononcent 'Beauchamp' Bitel, mais eux au contraire francisant à l'excès, comme feraient des paysans, appelant Mrs Bohnstone Mme Bonston» ${ }^{74}$. Et en effet dans le roman, un paysan prend le duc de Guermantes (alors prince des Laumes) pour «un English» ${ }^{75}$; plus haut, un autre prenait Robert de Léon pour «un Englische» ${ }^{76}$. Notons, pour conclure sur ce point, que ce langage

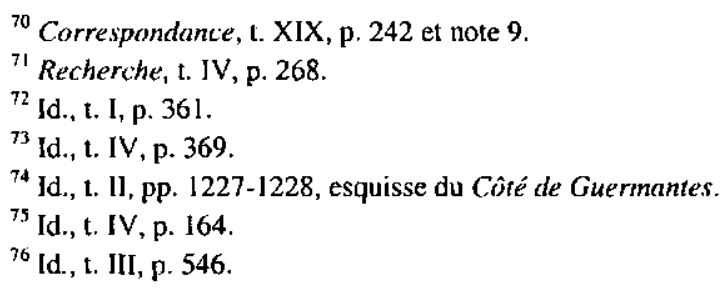


véhiculant les stéréotypes nationaux ne concourt pas seulement à donner du relief aux personnages; il illustre plus profondément la loi d'imitation inconsciente selon Tarde, lequel isolait dans le langage "le grand véhicule de toutes les imitations» ${ }^{77}$, nécessaire en effet à l'imitation «pour se répandre, par une sorte d'épidémie conquérante» ${ }^{78}$.

C'est par là que le stéréotype intéresse Proust, et finit par permettre paradoxalement une meilleure approche du phénomène artistique: il constitue une optique. Il est clair que ce qui retient l'attention du romancier mettant en scène un prince von ou un sculpteur Ski, c'est le perspectivisme qui en résulte. Un détail peut nous le suggérer: reçu pour la première fois chez les Guermantes, le héros est pris en charge par «un domestique qui attendait, vieux ou poudré, je ne sais, l'air d'un ministre espagnol, mais me témoignant du même respect qu'il eût mis aux pieds d'un roi» ${ }^{79}$. Cet exemple montre que le stéréotype national devient une curiosité précieuse quand il émane d'un artiste, parce qu'il ne réduit plus une personne à un type caricatural, mais illustre l'esthétique ici d'une école de peinture. Les questions de langage romanesque le suggéraient de leur côté, les stéréotypes nationaux sont aussi des stéréotypes littéraires: Proust refait, dans son pastiche sur l'affaire Lemoine, Balzac transcrivant la prononciation allemande du baron de Nucingen ${ }^{80}$; l'écrivain aime tout autant à retrouver des exemples de turqueries du XVII ${ }^{\mathfrak{e}}$ siècle: lisant en 1909 des extraits, donnés en avant-première dans Le Figaro, du livre de la princesse Bibesco Les huit Paradis: Perse - Asie Mineure - Constantinople, il écrit à l'auteur: «Je ne pense pas seulement à tout ce folklore oriental que vous inventez bien, je suppose, un peu, $[\ldots]$ mais à ces pages délicieusement peintes en turquerie $X V I^{e}$ siècle, où vous faites tout un décor, toute une atmosphère à un vers de Bajazet ${ }^{81}$.

Les rapports entre Balzac et la Pologne ravivent la question de l'optique propre à un écrivain. Dans la perspective anti-beuvienne de Proust, tout ce qui se dit sur le mariage de Balzac avec Mme Hanska illustre l'inanité de la méthode biographique. Ainsi, au cours des esquisses de la Recherche qui se forment - et pour cette raison sont classées - dans le chapitre sur Balzac de Contre Sainte-Beuve, la marquise de Villeparisis évoque le mariage polonais de l'auteur de La Comédie humaine comme une faillite de son entreprise littéraire: «Je l'ai vu une fois $[\ldots]$ quand j'étais toute jeune mariée, c'était un homme très commun, qui n'a dit que des choses insignifiantes, et je n'ai pas voulu qu'on me le présente. Je ne sais pas comment, sur la fin, il avait trouvé le moyen d'épouser une Polonaise d'une bonne famille qui était un peu parente à nos cousins Czartoryski. Toute la famille en a été désolée et je vous

\footnotetext{
${ }^{n}$ Les Lois de l'imitation, op. cit., p. 16.

${ }^{78}$ Ibid., pp. 368-369.

${ }^{79}$ Recherche, t. II, p. 715.

${ }^{80}$ Pastiches et mélanges, publiés avec Contre Sainte-Beuve et Essais et aricles par Pierre Clarac et Yves Sandre, Paris, Gallimard, «Bibliothèque de la Pléiade», 1971, pp. 9-10.

${ }^{81}$ Correspondance, t. IX, p. 241.
} 
assure qu'ils ne sont pas fiers quand on leur en parle. Du reste, cela a très mal fini. Et il est mort presque tout de suite» ${ }^{82}$. Cette mise en rapport, stupéfiante de naturel, entre le mariage mal assorti avec une Polonaise et l'inachèvement de La Comédie humaine, ouvre en fait à d'insondables mises en rapport entre la vie et l'wuvre d'un écrivain, à la faveur desquelles, comme bien souvent en coulisse, on ne sait plus nettement si Proust se situe pour ou contre Sainte-Beuve.

En effet, il faut d'abord savoir que la famille Proust était elle-même liée à la Pologne; c'est ce que révèle une lettre de juin 1915 évoquant, ce qui est rare sous la plume de l'épistolier, des souvenirs autobiographiques, à partir d'une mention de Clément de Maugny avec lequel l'écrivain est lié depuis 1899, et qui a épousé, en 1902, Rita Bussé, une jeune fille de père allemand et de mère polonaise originaire de Posen: «Clément était cousin des Krasynski et Czapski (excusez les fautes d'orthographe), d'où grandes conversations rue de Courcelles avec papa. Car je vous ai peut-être raconté que mon frère avait eu pour marraine une Comtesse Puslowska, née je crois, mais je n'en suis pas certain, Potocka ou peut-être Branicka, et dont le fils a épousé une Pignatelli. C'était une femme charmante qui nous couvrait de nécessaires de vermeil, et dont j'ai retrouvé une lettre dernièrement, que je trouve très gentille: elle demande à papa de venir voir la vieille bonne qui a élevé son mari et qui est très malade: 'Pardonnez-moi mon cher ami, de vous déranger pour elle, mais elle connaissait déjà Sigismond (son mari) quand je l'ai épousé, et il me semble qu'à elle aussi j'ai juré quelque chose'. Je ne sais pas si c'est le charme des souvenirs d'enfance, mais cela ne me semble pas mal. Lorsque Radziwill m'a parlé d'un comte Puslowski, 'le type du grand seigneur polonais', je n'ai pas osé lui demander de le connaître» ${ }^{83}$. Car ici, le personnage stéréotypé aurait été lié à des souvenirs familiaux.

À partir de là, Proust se livre à une transcription littéraire vertigineuse de la situation autobiographique, autour du cas de Balzac: en effet, ces considérations ont pour point de départ un faire-part annonçant le décès de la belle-fille de Balzac, avec laquelle la famille d'Alphonse Daudet était liée, la comtesse George Mniszech, née Anne Hańska (1828-1915): «Pour cette lettre Mniszech, à laquelle la vôtre (très enterrement de la duchesse de Langeais) confère toute sa beauté», Proust renvoie à la fin de son propre roman La Fugitive, annonçant le mariage de la nièce de Jupien, adoptée par le baron de Charlus, avec le jeune Cambremer, ce qui montre, ajoute le romancier, «qu'il suffit d'une seule alliance imprévue pour faire jouer tout le 'casus foderis' et défiler tous ces noms. Ce qui, d'ailleurs, là est faux, car à une certaine époque, toutes les grandes familles européennes s'allièrent (parfait défini très Balzac) aux Rzewuski, ... il me semble même que dans le Gotha il y a un Radziwił ${ }^{84}$ marié à Wirchownia (est-ce que ce n'est pas le nom de la propriété de Mme

${ }^{82}$ Contre Sainte-Beuve, éd. citée, pp. 283-284.

${ }^{83}$ Correspondance, $\mathrm{t}$. XIV, pp. 146-147.

${ }^{84}$ C'est, dans l'Almanach du Gotha, précisément à côté de la nolice Radziwil que se trouve celle sur la famille Radolinski-Radolin. 
Hańska?)»; il s'agit en fait de Wierzchownia en Ukraine et le prince Guillaume Radziwiłł (1845-1911) avait épousé en 1873 à Werchownia, gouvernement de Kiev, Catherine comtesse de Rzewuska.

Faisons le point: le mariage de Balzac avec Mme Hańska est mis en parallèle avec la marraine polonaise du frère de Proust; ce rapprochement nourrit une petite enquête sur les familles polonaises que l'on peut approcher à Paris, d'où il ressort que certaine peut être liée à Mme Balzac. Rejetant d'abord la méthode de SainteBeuve, le romancier de la Recherche s'assimile, par le biais de liens avec la Pologne, à celui de La Comédie humaine. Un mariage dans la société contemporaine conduit à un autre dans son roman; et une enquête dans le Gotha révèle que le tout pourrait bien remonter jusqu'à Balzac.

Tel est bien le stéréotype national pour un romancier: une banalité transformée en originalité par le véritable écrivain, qui la fera jouer dans son ouvre selon une certaine optique. Du côté de la banalité, par quoi tout ici commence, Proust a rencontré et loue, sous ta plume de son ami Jacques-Émile Blanche, «le parallèle entre les mours anglaises et françaises", dans les Cahiers d'un artiste qu'il lit en épreuves; Blanche écrivait: «Que de difficultés, de malentendus, d'incompréhensions, de flottement, avant qu'apprennent à se connaître nos deux peuples, aussi dissemblables l'un de l'autre que les Orientaux des Européens ${ }^{85}$. Chez Proust romancier, ce parallèle éculé devient cependant une question de perspectivisme: les qualités existent aussi chez les étrangers, rappelle le narrateur de la Recherche, mais avant de les apercevoir, «il faut d'abord traverser ce qui déplaît et ce qui choque et ce qui fait sourire» ${ }^{86}$. Or, c'est précisément cette traversée qui intéresse l'esthéticien; voilà pourquoi, au plus profond, il la mettra en scène de loin en loin dans son roman.

Mais dans ce type d'enquête, l'idéal à atteindre se situe en dehors du champ du roman - dans la poésie. Seule la poésie en effet, si elle est pure image, peut transfigurer en un instant le stéréotype le plus éculé en la vision la plus neuve. La condensation poétique de tous ses efforts de romancier mettant en scène des personnages multinationaux, Proust l'a trouvée chez un poète contemporain, dans le poème Les voyages inséré par Anna de Noailles dans L'Ombre des jours (1092), et dont l'épistolier se plaît à citer cette strophe:

Voir la Chine buvant aux belles porcelaines,

L'Inde jaune accroupie et fumant ses poisons,

La Suède d'argent avec ses deux saisons,

Le Maroc en arceaux, sa mosquée et ses laines.

Combien de conversations stéréotypées, sur ces diverses contrées du monde, que développera tout au long un roman, viennent former ici un chapelet de précieux sulfures, chaque alexandrin enfermant l'essence d'un univers lointain dans son arrondi de verre.

\footnotetext{
${ }^{85}$ Correspondance, t. XIV, p. $157 \mathrm{ct}$ note 12.

${ }^{86}$ Recherche, t. II, p. 702.
} 